\title{
Special Issue: Imaging of gastrointestinal and urogenital tracts
}

\author{
Rick R. Van Rijn
}

Received: 12 November 2010 /Accepted: 12 November 2010 /Published online: 4 December 2010

(C) The Author(s) 2010. This article is published with open access at Springerlink.com

This special issue focuses on imaging of the gastrointestinal and urogenital tracts; the papers were initially presented at the European Course of Paediatric Radiology (ECPR) in 2009 in Amsterdam, the Netherlands. The ECPR is an annual course with four revolving topics, i.e. chest/ cardiovascular, musculoskeletal, neuroradiology (in collaboration with the European Society of Neuroradiology and the European Society of Magnetic Resonance in Neuropediatrics) and the gastrointestinal/urogenital tract. The aim of this annual course is to enhance knowledge in paediatric radiology; both focussed at fellows in paediatric radiology as well as established radiologists with a special interest in our discipline. It also serves as a meeting point at which collegial and social bonds across borders are formed and enhanced.

Paediatric radiology of the gastrointestinal and urogenital tracts has seen a significant change in clinical practice over the last decades. Although as a result of close patient contact, our discipline always has been and hopefully will remain a highly clinically involved field of radiology, changes in diagnostic strategies have changed our daily practice. In many paediatric radiology departments today the fluoroscopy rooms are unoccupied most of the time and barium, in many cases, has been replaced by gadolinium.

The purpose of this special issue is to provide an upto-date overview of imaging of the gastrointestinal and

\section{R. R. Van Rijn $(\triangle)$}

Department of Radiology, Academic Medical Center Amsterdam, Meibergdreef 9,

Amsterdam 1105 AZ, Netherlands

e-mail: r.r.vanrijn@amc.uva.nl urogenital tracts. Apart from the use of fluoroscopy, which in selected cases is still very valuable as a diagnostic tool, the use of CT and especially MRI is discussed. The use of MRI has taken off and there are promising developments that make the application of this technique an increasingly realistic diagnostic modality in the field of paediatric radiology. In addition to imaging of anatomical structures, MRI can be used to measure function. Using dedicated software (available as freeware) parameters such as renal transit time and differential renal function can be calculated. This makes MRI a one-stop-shop imaging technique for renal diseases. In abdominal imaging the application of diffusion-weighted imaging (DWI) and diffusion-weighted whole-body imaging with background signal suppression (DWIBS) opens new frontiers where anatomical and functional imaging are combined in one modality.

Another exciting development is the implementation of interventional radiology. Along with the development of minimally invasive surgery, interventional radiology is gaining ground within paediatrics. The acceptance however, has not reached a level comparable to that in adult medicine, where interventional radiology has a strong position and interventional radiologists are seen as an important asset to any radiological department. In two papers the application of gastrointestinal and urogenital interventional radiology is discussed; it is hoped that these papers will aid further development and implementation of these techniques in paediatric radiology.

The combination of close patient contact, the daily pleasure of working with children and their parents and rapid implementation of state-of-the-art imaging modalities makes paediatric radiology a unique subspecialty. It has 
been shown that the use of advanced/multimodality imaging and intellectual challenges in the USA are among the top reasons for choosing a subspecialty career. I believe that paediatric radiology offers both and it is therefore surprising to see that throughout Europe and the USA there is still a relative shortage of paediatric radiologists.

As an editor of this special issue, and on behalf of my co-organisers Mrs. A.M.J.B. Smets and Mrs. E.E. Deurloo,
I would like to thank the faculty of the 2009 ECPR for their contribution to the education of a new generation of paediatric radiologists.

Open Access This article is distributed under the terms of the Creative Commons Attribution Noncommercial License which permits any noncommercial use, distribution, and reproduction in any medium, provided the original author(s) and source are credited. 